\title{
Toward the Verification of a Simple Hypervisor
}

\author{
Mike Dahlin, Ryan Johnson, Robert Bellarmine Krug, Michael McCoyd, William Young \\ Department of Computer Sciences \\ University of Texas at Austin \\ \{dahlin, rjohnson, rkrug, mccoyd, byoung\}@cs.utexas.edu
}

\begin{abstract}
Virtualization promises significant benefits in security, efficiency, dependability, and cost. Achieving these benefits depends upon the reliability of the underlying virtual machine monitors (hypervisors). This paper describes an ongoing project to develop and verify MinVisor, a simple but functional Type-I x86 hypervisor, proving protection properties at the assembly level using ACL2. Originally based on an existing research hypervisor, MinVisor provides protection of its own memory from a malicious guest. Our long-term goal is to fully verify MinVisor, providing a vehicle to investigate the modeling and verification of hypervisors at the implementation level, and also a basis for further systems research. Functional segments of the MinVisor C code base are translated into Y86 assembly, and verified with respect to the Y86 model. The inductive assertions (also known as "compositional cutpoints") methodology is used to prove the correctness of the code. The proof of the code that sets up the nested page tables is described. We compare this project to related efforts in systems code verification and outline some useful steps forward.
\end{abstract}

\section{Introduction}

Platform virtualization refers to technologies that provide a layer of abstraction between computer systems and the operating systems that utilize them. It has existed for many years and appeared in various guises, but has recently emerged as a key technology with vast potential impact on commercial and government computing systems. Platform virtualization promises significant benefits in security, efficiency, dependability and cost [11]. Achieving these benefits depends upon the reliability of the virtual machine monitors (hypervisors) that provide them.

We believe that providing high assurance of the properties of a system as complex as a hypervisor requires a rigorous formal analysis, stretching from the desired security properties at the top, down to the physical hardware and implementation code at the bottom. This project aims to develop a simple but useful hypervisor and to provide very high assurance in its properties by using ACL2 [15] to verify the hypervisor implementation.

For the purposes of this paper, a hypervisor, also called a virtual machine monitor (VMM), is a software system that virtualizes some system resources of the host computer and makes them available to one or more guests. Hypervisors provide a range of functionalities [26] and a diversity of guarantees. We chose to pursue verification of a hypervisor because of the increasing impact of hypervisors on commercial and government systems. However, existing commodity hypervisors have a large, complex and changing code base and seem beyond the reach of current modeling and verification techniques. Instead, we focused on the verification of a small research hypervisor that we could envision tackling with the limited resources available to the project, in hopes that the tools and techniques developed might scale to larger system software verification efforts.

Though our target hypervisor, called MinVisor, runs only one guest, its features provide some of the minimum requirements of typical hypervisors and provide some of the guarantees of CMU's SecVi- 
sor [25], a simple research hypervisor that we used as our initial jumping off point. Thus, MinVisor provides a simplified but useful platform on which to carry out our initial verification efforts.

This paper reports on the development of MinVisor and progress in its modeling and verification. Though early, the project has had some significant success in moving toward using formal verification to develop a simple hypervisor with very high assurance.

In Section 2 we describe the overall strategy of the project. Section 3 outlines the hypervisor artifact we are constructing. Section 4 describes the Y86++ model on which our proofs are constructed. In Section 5] we outline our proof strategy and describe the proof of specific critical MinVisor functionality. Section 6 lists some relevant related work. Finally, in Section 7 we outline our plans for extending this effort. The Appendix contains the $\mathrm{C}$ source code and Y86++ assembly level code for specific MinVisor functions.

\section{High Level Strategy}

The overall goal of this project is to investigate techniques for modeling and verifying a simple hypervisor. At the end of the day, we hope to demonstrate that:

1. We can carry out the verification of a hypervisor at a very low level of abstraction to provide high assurance of correctness.

2. The resulting verified artifact is a credible piece of system software displaying useful hypervisor functionality.

3. The tools and techniques we develop will scale to apply to more realistic and full featured hypervisors.

Modeling and verification of a complex artifact might follow a top-down approach-taking an abstract model of the domain (hypervisors in this case) and refining it toward a compliant implementation. Alternatively, it might follow a bottom-up approach-extracting and proving properties of implementation level modules with the goal of combining these into a coherent verified model of the complete artifact. A hybrid approach is probably best, to ensure that the two "meet in the middle."

To date, we have worked largely bottom up, concentrating on developing the techniques necessary for verifying the many lines of machine code that comprise the MinVisor code base. We felt that defining a reasonable machine model and building the libraries and infrastructure to allow efficiently proving significant fragments of code was probably our biggest hurdle. Consequently, the description of the modeling and verification below is focused on the low level. We describe the verification of the code that sets up MinVisor's nested page tables. This functionality is critical to allow MinVisor to protect itself from a malicious guest, and seems representative of the code complexity we will encounter moving forward. We believe that this or very similar code will be required for any hypervisor.

Our initial code base was extracted from CMU's SecVisor's code [25]. However, we made various simplifications to eliminate functionality orthogonal to our concerns and to simplify our initial proof target. For example, SecVisor is tightly coupled with its modified Linux guest, making Linux part of the trusted computing base. By making various modifications we were able to eliminate this dependency, yielding a simpler code base to verify and a more broadly applicable hypervisor. MinVisor loads the guest as if by the BIOS and protects the hypervisor state via nested paging.

Protection of the core system and software is crucial to any system making security claims, and is the primary initial goal of this project. However, in order to be useful, hypervisors must also provide a reasonable operating environment to the guest. Our current work does not perform any sort of corrective 
emulation, nor any paravirtualization to provide alternate resource access. The unmodified guest runs freely as long as it performs only "safe" actions. Dangerous operations are intercepted and prevented, but MinVisor's operations when doing so may be detectable by the guest. Providing a reasonably transparent and accurate simulation of a physical machine is future work.

\section{MinVisor}

MinVisor was developed to examine the proof of the protection of a hypervisor and machine from a malicious guest and as a base for further systems work. It is a minimal Type-I/bare-metal hypervisor for the $\mathrm{x} 86$ utilizing AMD's hardware assisted AMD-V nested page table virtualization. The hardware BIOS loads MinVisor from the network using the standard BIOS PreBoot Execution Environment (PXE) [8]. MinVisor loads the first hard drive sector, as if by the BIOS, as the guest. On the QEMU x86 emulator it runs a real and protected mode guest that tries to probe memory in each $2 \mathrm{MiB}$ machine page. It has not been tested on real hardware. MinVisor is written in a mix of assembly and C, limited to 32 bit instructions, and draws from code in SecVisor, JOS [14], and Xen [3].

MinVisor was designed as a real hardware, tiny, hypervisor that does the essential tasks of any AMD$\mathrm{V}$ nested paging hypervisor as simply as possible. These essential tasks are:

- Create host save area and VMCB,

- Create IO and MSR permission bit maps,

- Set guest actions to intercept,

- Populate nested page tables,

- Set starting guest state in VMCB,

- Load guest code,

- Populate and activate host page tables,

- Start the guest, and

- Handle intercepts (for nested page faults).

Except for status messages and memory read values (below) MinVisor currently does only these things.

Though MinVisor started from SecVisor, we have made many simplifications and the code is now closer to KVM [17] in organization. We have focused on providing absolute protection of the host and hardware from an arbitrary and potentially malicious guest. Secondarily, we provide some transparency to the guest by booting over PXE which leaves no hard drive evidence of our existence. Our initial phase aims to protect MinVisor's memory. If MinVisor's memory is not protected, no guarantees can be made about its operation.

MinVisor is the first code to run after the BIOS, whose PXE tftp code downloads MinVisor to physical address 0x7C00. Control is transferred to MinVisor in real mode, and MinVisor makes no further use of the BIOS PXE tftp capabilities. After relocating itself to the highest available 2MiB aligned memory chunk and establishing protections, MinVisor emulates the behavior of the BIOS had PXE not been called, and copies the first sector from the hard disk to $0 x 7 \mathrm{C} 00$, acting as a real mode boot loader. We assume a static root of trust of the boot path. The guest is currently limited to 4GiB of RAM.

MinVisor emulates a normal system startup with its real mode start of the hard drive's boot sector as the guest. This minimizes interdependencies between MinVisor and the guest. MinVisor need not receive any information from the guest, and the guest need not be aware that MinVisor is running. It 
should allow any hard drive bootable guest to be run, and it allows MinVisor to run on any machine with PXE, which is widespread in both consumer and enterprise hardware. Booting MinVisor with PXE allows providing the entire hard drive to the guest, avoiding the need to virtualize it.

Most control over the guest is exercised through proper configuration of the virtual machine control block (VMCB), a per-guest memory region maintained by the hypervisor and virtualization hardware that contains settings and information necessary to launch a guest through AMD-V mechanisms. The VMCB points to the nested page table that provides protection from direct access to MinVisor's own memory.

Normally, process effective addresses are translated to physical addresses through segmentation or segmentation with paging. First, the addresses are combined with the appropriate segment register to create a linear address. If page tables are used, this linear (or virtual) address then serves as the index into the page tables and a physical address is obtained. It is this physical address that is used by the hardware.

However, a third translation is involved for guest addresses if hardware virtualization is using nested paging. The guest physical address obtained by the first lookup is used to index into the nested page tables, and a true system physical address is obtained. These nested page tables are used by MinVisor to protect its memory regions, and are more fully described in Sec. 4.1 .

Except for a few regions, MinVisor's nested page tables establish an identity map from guest "physical" to system physical memory; most addresses in the guest will resolve to the same location as they would if the guest were running on the bare machine. The identity map was chosen for simplicity, though a more complex mapping would have been only marginally more difficult to implement and verify. The exception to the mapping is that addresses in the top $2 \mathrm{MiB}$ of physical memory have an empty "not present" mapping from the guest. Thus, no guest address will be translated to the physical memory where MinVisor is located.

Should the guest, through ignorance or malice, try to access an address marked "not present" in the nested page tables, the AMD-V hardware will generate an intercept and return to host mode with information about the guest action. In such cases, the MinVisor intercept handler causes writes to protected regions to be no-ops, and reads return bytes from within the 16 byte string "Th Eyes of Texas". Use of the hardware architecture's nested page tables greatly simplifies MinVisor and its proof, and allows the guest to run uninterrupted most of the time. To help the guest use reliable memory, we virtualize the BIOS memory map to report the top of physical memory as $2 \mathrm{MiB}$ lower than it actually is.

Interception of guest access to hardware $\mathrm{I} / \mathrm{O}$ is restricted via a table referenced in the VMCB. We currently do not intercept any hardware ports, but plan to default to intercepting all of them. This interception requires some emulation of functionality in the intercept routines in order to provide required $\mathrm{I} / \mathrm{O}$ services to the guest.

The VMCB also contains a bit map allowing selective interception of various operations. We currently intercept all operations related to interrupts, control registers, and virtualization and convert them to no-ops. In order to provide a reasonable operating environment to a non-trivial guest, we will need to emulate or forward basic interrupt handling and some system control events.

Initially we are implementing paranoid protection from DMA enabled devices. Rather than intelligently map and cordon devices, all devices are prevented from writing to the location of MinVisor or to memory mapped I/O regions. This satisfies our initial protection and code simplicity goals, with performance and functionality issues to be addressed later.

Though MinVisor is sparsely featured by combination of design and its early stage of development, we feel it is still a reasonable hypervisor target for a proof of implementation. The core memory protection mechanisms it provides by way of hardware initialization and software routines are necessary 


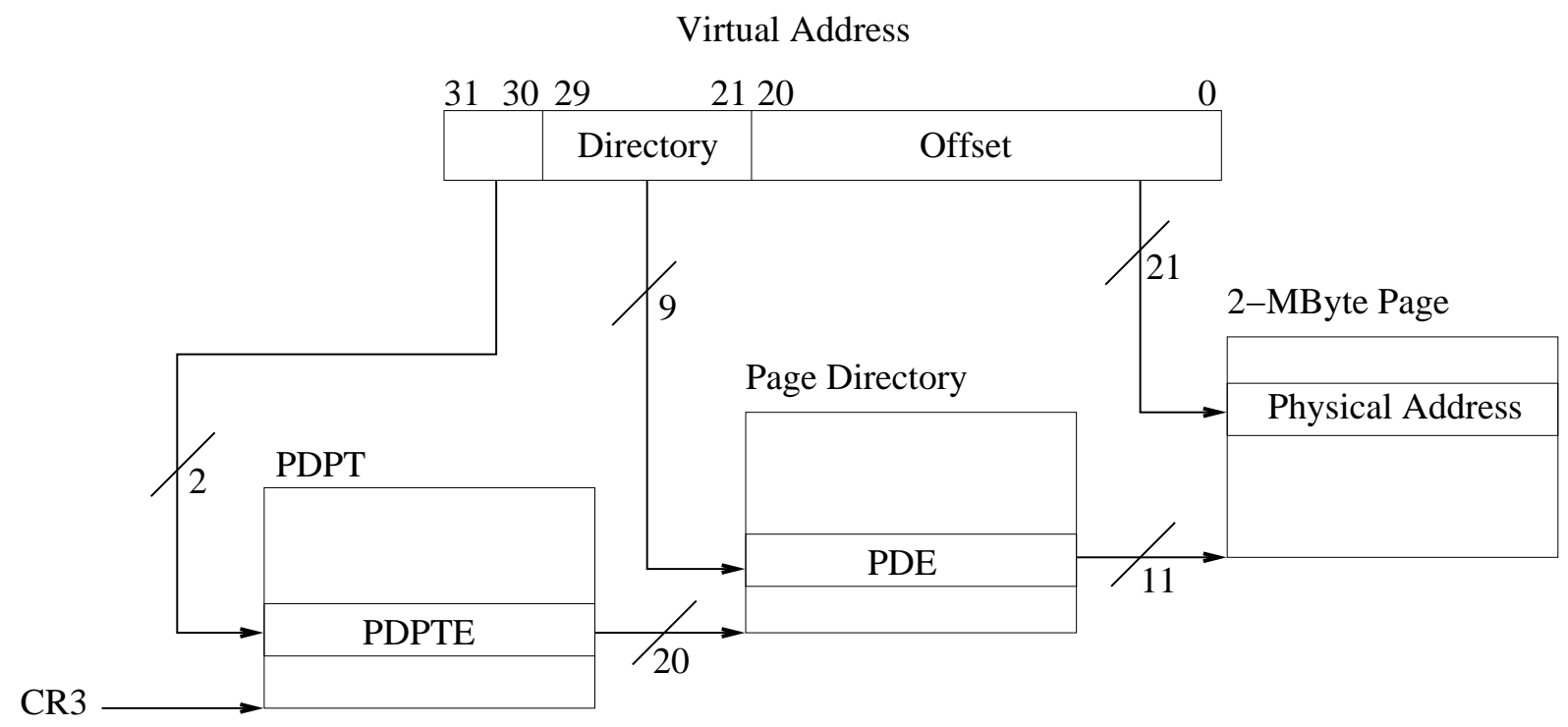

Figure 1: Linear Address Translation to a 2-MiByte Page

functionality for any hypervisor. Additional features and functions will build on top of the initial code and proof, rather than supplant it.

\section{The Y86++ Model}

The goal of this project is a simple hypervisor verified at the implementation level. This requires a formal model of the implementation language, and the machine it runs on. Several related efforts [16, 27] choose to formalize subsets of $\mathrm{C} / \mathrm{C}++$. We opted instead to conduct our proofs at the machine code level. This choice eliminates relying on any formal model of $\mathrm{C} / \mathrm{C}++$ and capitalizes on strengths of ACL2. But ideally, it means verifying code against a formalization in ACL2 of the x86 instruction set architecture. Though colleagues are working on such a model, it has not reached a stable and usable state.

To allow progress on this project, we began our proofs instead on a modified version of Bryant and O'Hallaron's Y86 model [6]. The Y86, designed for pedagogical purposes, is a much simplified version of the $\mathrm{x} 86$ instruction set architecture. The Y86 was originally formalized in ACL2 by Sandip Ray [23] for his dissertation work. Another formalization in ACL2 was developed by Warren Hunt for use in his computer architecture classes at the University of Texas at Austin.

Over the course of this project, we have modified Hunt's Y86 model by adding several components to the processor state, several new instructions, a guest/supervisor mode flag, and nested page tables. In this paper, we refer to our extension of the Y86 model as the Y86++, to prevent confusion with either Bryant and O'Hallaron's Y86 or Hunt's implementation of it in ACL2. Because of the similarity of the Y86++ to the $\mathrm{x} 86$, we believe that the verification work we have done to date will translate rather directly to an x86 model when one becomes available. The proof techniques and invariants are generally independent of the details of the Y86++. 


\subsection{Changes from Y86}

The Y86 is an instruction set architecture in the style of the x86. We refer the interested reader to [6] for details. We have made the following additions to Bryant and O'Hallaron's Y86:

- Two new registers IMME1, VALU1: used as "scratch registers" to more closely emulate specific complex x86 instructions as a series of Y86++ instructions. Examples of their use will be given in Section 4.2

- A register CR3: points to the base of the page table hierarchy.

- A carry flag: used in several of the new instructions.

- A 1-bit Guest/Supervisor Mode flag, and four 32-bit registers: these allow mode switching and preservation of ESP and EIP when switching modes.

In addition, we have added the following instructions:

- adcl (add with carry),

- cmpl (compare),

- sall (arithmetic shift left),

- shrl (shift right),

- jb (jump if below),

- jbe (jump if below or equal).

Memory in the Y86++, as in Hunt's implementation of the Y86, is modeled as an association list from 32-bit addresses to 8-bit values. We have implemented a nested page table mechanism on top of that. Paging is enabled only when the Y86++ is in Guest Mode.

The Y86++ paging mode is modeled on Intel's PAE paging, which is what is used by SecVisor and MinVisor for the nested page tables. With PAE paging (see Figure 1), the first paging structure comprises only 4 entries and is pointed to by the CR3 register. Translation uses bits $31: 30$ from a 32-bit virtual linear address to index the appropriate entry. This entry points to the appropriate second level paging structure, comprising $512=2^{9}$ entries. Bits $29: 21$ identify the appropriate entry pointing to a $2 \mathrm{MiB}$ page frame. Both entries also contain certain bits identifying the status of the page frame. The only status bit we concern ourselves with at the moment is the page present bit. If this bit is 0 (as is the case for all pages that MinVisor protects), a page fault is thrown if the page is accessed. At the moment, the Y86++ does not properly handle page faults. This is not relevant to the theorems we have proved, but will be relevant in subsequent work.

We have implemented an assembler in ACL2 to convert Y86++ assembly language programs into their binary format, based on Hunt's Y86 assembler. This is merely a convenience. Our proof is at the level of the binary; its validity does not depend on the assembler.

\subsection{Comparison to the $x 86$}

The Y86++ assembly is similar to a restricted subset of the $\mathrm{x} 86$. Other than the limited number of instructions modeled, the main restriction is in the handling of immediate values - the only instruction that handles these is the irmovl (immediate to register move) instruction.

The following is a fragment of Y86++ assembly generated by hand from the corresponding $\mathrm{x} 86$ instructions (in the comment column). The $\mathrm{x} 86$ assembly was generated by the gcc compiler from a portion of the MinVisor implementation. 


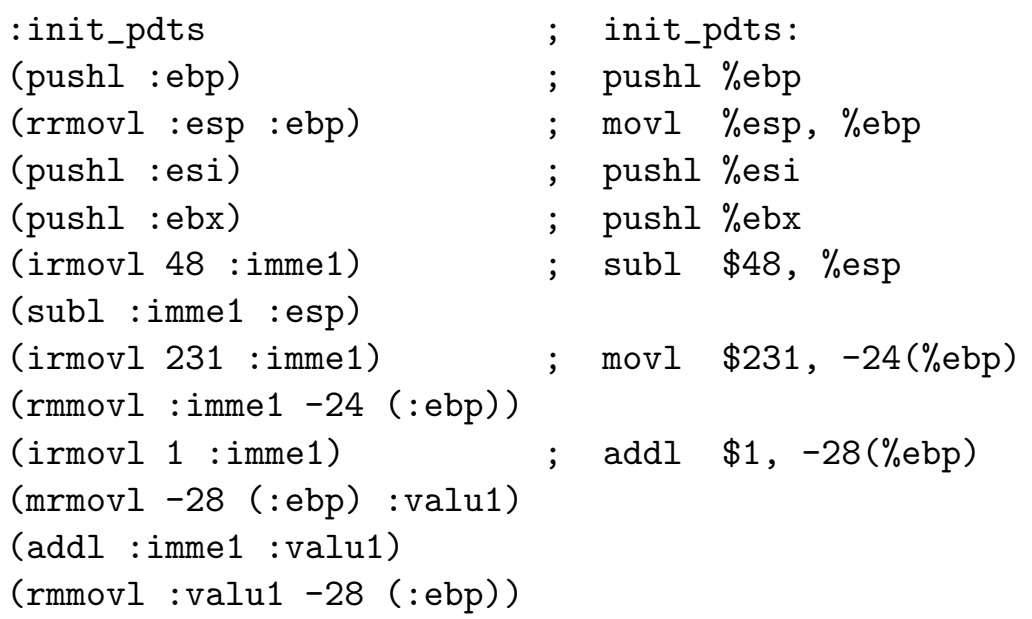

Note that many of the statements translate directly. The more complex translations involve instruction modes (using immediate values) not present in the Y86 or Y86++ models. For example, subtracting an immediate value from a memory location (e.g., sub1 $\$ 48, \%$ esp) requires moving the immediate value into a register first, since only immediate to register operations are modeled.

In each case, the translation has been crafted to match the semantics of the corresponding $x 86$ instruction. The addition of the : imme 1 and : valu1 registers makes this translation both simple and direct. We believe that the resulting state of the Y86++ memory and the state of all registers and flags that correspond to $x 86$ structures are identical to what follows the execution of the original x86 instructions. That claim has not been formally established, however, and cannot be until we have an x86 model in hand. When we do, we plan to switch our proof effort to the $\mathrm{x} 86$ model, and this question will become moot.

\section{Implementation Level Proofs}

MinVisor code is verified following the "cutpoints" approach described elsewhere [19, 22]. Given an operational semantics for a machine language and an annotated program, this allows the mechanized generation of verification conditions adequate to establish the partial (or total) correctness of the code. ACL2 is then used to dispatch the verification conditions.

The method is compositional, in that the correctness of a subroutine needs to be proved once, rather than at each call site. The method has been used to verify several machine-level programs prior to the current project using the ACL2 theorem prover.

To date, we have completed proofs of selected portions of MinVisor codebase. In particular, we describe below a proof of the functions that set up the MinVisor nested page tables. Those pages that MinVisor wishes to protect-its own memory image and the nested page table structures-are marked as not present. Address translation for the rest of memory is the identity map. The process of setting up the page tables is described in the following subsection. Then in Section 5.2 we give a description of the ACL2 modeling and proof.

\subsection{Correctness of the Page Table Code}

In our Y86++ model, the function (va-to-pa addr $\mathrm{s}$ ) is responsible for the translation from a virtual address addr to a physical address via a page table lookup in the state s. If paging is turned off, va-to-pa reduces to the identity map on addr. If paging is turned on, address translation is done via the 
page tables as indicated in Figure 1. Normally, this will result in va-to-pa returning an address. But, if during this lookup the entries used contains a page present bit set to 0 , a page fault is raised.

The $\mathrm{C}$ function create_nested_pt (actually the corresponding Y86++ binary) is responsible for setting up the nested page tables to be used by MinVisor. The appendix contains the $\mathrm{C}$ source code and a sample of the corresponding Y86++ assembly level code. create_nested_pt calls three subsidiary functions: init_pdpt sets up the top level Page Directory Pointer Table; init_pdts sets up the four second level Page Directory Tables; sec_not_present zeros out those entries, thereby marking those pages as not present. The memory region to be protected is indicated via parameters passed to create_nested_pt giving the start of the memory region to be protected and its size.

Let SO be the initial state before create_nested_pt is run. Let S1 be the state after it has run to termination, the CR3 register point to the top of the nested page tables, and assume paging has been turned on. Our final top-level theorems state that the nested page tables set up by MinVisor are indeed the desired page tables.

More specifically, we prove the following two facts:

- If the precondition holds for $\mathrm{S} 0$ and addr is disjoint from the memory region to be protected, then (va-to-pa addr $\mathrm{S} 1$ ) returns addr.

- If the precondition holds for $\mathrm{S} 0$ and addr is not disjoint from the memory region to be protected, then (va-to-pa addr S1) signals a page fault.

The precondition states that:

1. The initial Y86++ state is well formed;

2. The code is loaded at a specified location in memory;

3. The machine is poised to execute create_nested_pt

4. Paging is turned off on the machine;

5. The code, stack, and the tables being created are located at disjoint memory locations;

6. The tables are aligned on $4 \mathrm{~K}$ page boundaries

7. The start of the memory region to be protected is aligned on a $2 \mathrm{MiB}$ page boundary;

8. The size of the memory region to be protected is a non-zero multiple of $2 \mathrm{MiB}$;

9. The stack does not wrap around memory;

10. All memory addresses used fit into a 32-bit register.

\subsection{The ACL2 Model}

In this section, we describe the modeling in ACL2 of the theorems proved about init_pdts. This function initializes the four page directory tables, and our theorem specifies its effects. It is formalized using the cutpoints method described above.

The $\mathrm{C}$ code for the proved functionality is listed in Appendix A. This is compiled using the gcc compiler into $\mathrm{x} 86$ assembler, which is hand translated into Y86++ code. The resulting Y86++ code for the init_pdts function is listed in Appendix B. This hand translation could be automated, but we have not done so because we hope to evolve our verification process to handle real x86 code. However, as illustrated in section 4.2 above, the translation is straightforward, so does not introduce significant uncertainty into the overall process. The Y86++ code is then assembled into a Y86++ binary, and it is the binary that is the target of verification. 
The pre-condition of the execution (the 10 conditions listed in the previous section) is modeled by an ACL2 predicate INIT_PDTS-PRE on the initial state. The results of the symbolic execution are modeled by the ACL2 function INIT_PDTS-MODIFY specifying the resulting state as a series of modifications to the initial state. This specifies all changes to registers, flags, memory and other system components.

This result function is tedious to construct and error-prone. At some point we hope to use "wormhole abstraction" [10] to elide state changes that are not of interest. We believe this will simplify these theorems considerably.

Alternatively, with some modifications to the macros used to generate the theorems, we could specify a series of "read over write" theorems as follows. Let SO be the initial state before the code being analyzed is run and $\mathrm{S} 1$ the state after the code is run to termination. We can construct:

1. A frame condition, specifying that reading in S1 those portions of state not changed by the code, can be simplified to an equivalent read in $\mathrm{S} 0$.

2. Theorems specifying, for those portions of state that are changed, how a read in S1 would be reduced to reads in $\mathrm{S} 0$.

This could be used to replace the effects function.

The theorem we currently prove shows that running the machine on a suitable initial state yields a resulting state identical to the initial state except for specific concrete changes.

As with all ACL2 cutpoint proofs, the user supplies: the definition of the language interpreter (in this case the Y86++ model), the pre-conditions of the code's execution, and the expected modification to the state. Code involving loops also requires that appropriate loop invariants be given. A series of theorems are generated that, if proven, are adequate to show that a terminating execution of code from an initial state satisfying the precondition will produce the indicated result. Matthews, et al. [19] developed ACL2 macros that provide a convenient syntactic framework for supplying the components and generating the requisite theorems. See [19] for a thorough description of the methodology.

The following is the macro call that generates the forms submitted to ACL2 in the proof of the function.

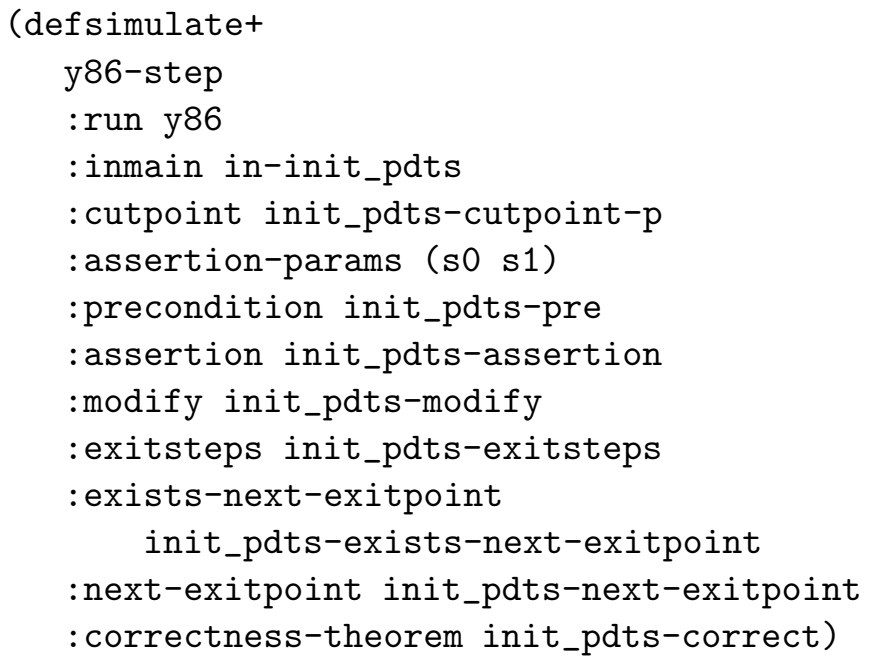

Executing this form generates a complex ACL2 encapsulate that culminates in the following theorem:

(DEFTHM INIT_PDTS-CORRECT

(IMPLIES

(AND (INIT_PDTS-PRE S1) 


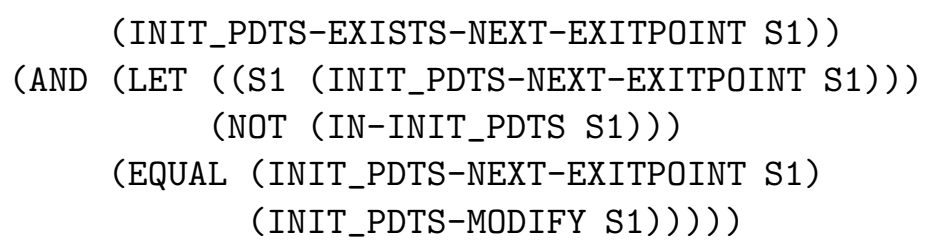

This theorem is proved mechanically by ACL2. Developing the proof, however, did require the development of many subsidiary lemmas and enhancements to the underlying libraries about our Y86++ model. Specifically, we developed a library for specifying and reasoning about disjoint memory regions. We expect that this effort can be amortized over many such proofs on the MinVisor project.

We have not yet considered termination in this proof, but believe that that can be trivially added for the fixed finite structures we are dealing with.

The cutpoints methodology has been used previously [19] in the verification of a JVM implementation of an encryption/decryption system. The MinVisor proofs may be the first sizable application stressing this technology; we anticipate the need to verify thousands of lines of binary code.

\section{Related Work}

Hypervisors were first introduced in the 1960s as a way to multiplex scarce and expensive computing resources. The advent of inexpensive hardware and multitasking operating systems eroded their value in the 1980s and 1990s. Over the last few years, however, hypervisors have regained popularity as a versatile technology for enhancing security and reliability. One effect is that processor manufacturers are removing obstacles that made it difficult to virtualize some system resources on earlier processor designs without significant emulation performance penalties. Both Intel and AMD have developed virtualization extensions to the x86 architecture [1, 13].

Xenon[20, 21] is a high assurance hypervisor based on re-engineering Xen. The designers have specified a formal security policy based on the notion of independence [24]. Though related to our work, the Xenon hypervisor is much larger (around 70,000 lines of code) and the effort is focused on gaining assurance through a policy-to-code development methodology. In another large effort, Microsoft has developed and used VCC [7], A Verifier for Concurrent C, to verify components of their 60,000 line Hyper-V hypervisor.

Several research efforts have demonstrated that it is possible to construct small, robust and useful hypervisors. The SecVisor project [25] implemented two hypervisors (1739 and 1112 lines of code, respectively) supporting Linux kernel version 2.6.20. Their systems provide strong integrity guarantees. However, a subsequent formal analysis [9] using the Mur $\phi$ model checker found two significant and exploitable design flaws.

Various projects have tied together hardware and software verification. The CLI Stack [4] was a collection of verified components including a simple high-level language compiler, a linking loader, and a microprocessor model. The European Verisoft project [28] has taken a similar approach with the goal of "pervasive formal verification" of an entire computer system including hardware (processor and devices), a real-time operating system, and applications. This effort has since evolved into the Verisoft $\mathrm{XT}$ project and consortium.

The Fiasco project developed a microkernel running on x86 PCs and intended to be compatible with the L4 microkernel. The related VFiasco effort attempted to prove security properties from the Fiasco source code including a small hypervisor developed in C++. A "semantics compiler" translates the $\mathrm{C}++$ 
code into logical formulas that are analyzed using PVS. They also use a formal but incomplete x86 model. [12]

One component of the Robin Project [27] from Radboud University Nijmegen in the Netherlands aims to verify the Nova hypervisor, a micro-hypervisor targeted at the $\mathrm{x} 86$ architecture, using a very similar approach to the VFiasco work.

A collaboration between NICTA, UNSW, Open Kernel Labs, and ANU resulted in the creation and verification of seL4 [16], a third-generation L4 family microkernel. Their proof is done using the Isabelle/HOL theorem prover, and involves proof of refinement across three layers-an abstract specification, a functional prototype in subset of Haskell, and an implementation in a subset of C.

Other projects have attempted to apply formal methods to establish certain separation properties. Leslie, et al. [18] have developed a small microkernel/hypervisor in Haskell and specified a combination of noninterference and information flow properties. This, they claim, provides a first step towards a formally verified separation kernel, and reduces the gap between information flow theory and operating system practice. Alkassar and Paul [2] report some initial work toward the verification of a "baby" hypervisor for the DLX RISC architecture. A related effort is the VeriOS project of the Saarland University (Germany). Its goal is the verification of an L4 kernel running on their own verified microprocessor VAMP [5]. The processor is modelled down to the gate level using NuSMV in conjunction with Isabelle/HOL.

\section{Conclusions and Future Work}

This project is a collaboration between the Systems and ACL2 groups at the University of Texas at Austin. As a result, our efforts have been focused in two tracks:

1. Constructing the MinVisor hypervisor;

2. Developing the modeling and proof infrastructure to permit the formal verification of MinVisor.

We have found this to be a highly useful collaboration in that systems expertise was necessary to ensure that the artifact we built is credible from a systems standpoint. Our expertise in using ACL2 gave us hope that we could manage this complex verification effort.

Our goal has been to construct a simple but realistic hypervisor with the goal of proving its correctness at the implementation level. Though the effort is far from complete, we can report significant progress, including:

- Building a simple, running hypervisor called MinVisor that protects itself from the guest;

- Enhancing the formal Y86 instruction set model to be able to encode critical MinVisor functionality;

- Developing proof techniques adequate to prove the correctness of that functionality.

A project concern was to find a balance of a useful hypervisor and one we could verify. It is too early in the project to have definitive evidence that our hypervisor is both credible and verifiable. By running on real hardware, we aimed to make the artifact interesting from the systems perspective. But our first cut is quite simple compared to commercial hypervisors and even compared to SecVisor. However, our goal is to augment MinVisor with additional functionality once we have developed sufficient proof infrastructure and facility doing the proofs to convince ourselves that the project will succeed.

On the systems side we hope to: 
- Virtualize a small critical set of devices (e.g. console, timer, network) and block access to other devices.

- Evolve MinVisor to support multiple guests.

- Enhance CPU virtualization to multiplex among guests.

- Enhance the fidelity with which the guest thinks it has real hardware.

On the verification track, we hope to do the following:

- Move from the Y86++ model we are currently using to an x86 model as one becomes available.

- Prove rigorously the entire MinVisor code base.

- Make use of new ACL2 proof techniques to reason more efficiently about assembly code.

As we noted in Section 2, we hope to demonstrate that:

1. We can carry out the verification of a hypervisor at a very low level of abstraction to provide high assurance of correctness.

2. The resulting verified artifact is a credible piece of system software displaying useful hypervisor functionality.

3. The tools and techniques we develop will scale to apply to more realistic and full featured hypervisors.

Our progress to date makes us hopeful that we can achieve these goals. However, it is too early to claim success on any of them.

\section{Acknowledgements}

We thank our colleagues at the University of Texas, particularly Sandip Ray, Warren Hunt, and J Moore for their work on the Y86, and Matt Kaufmann for help with ACL2. We'd particularly like to thank Sandip Ray for helpful discussions throughout the project and comments on the draft of this paper. The comments from the anonymous reviewers were also very helpful. This material is based upon work supported by the National Science Foundation under Grant No. CNS-0917162, and by Raytheon under contract 200901296.

\section{Appendix}

\section{A C Source Code}

We here give the $\mathrm{C}$ source for that part of MinVisor that sets up the nested page tables. We analyzed the corresponding Y86++ binary code.

typedef unsigned int u32;

typedef unsigned long long u64;

// pointer to the page-directory-pointer table

typedef u64 *pdpt_t;

// pointer to a page-directory table 


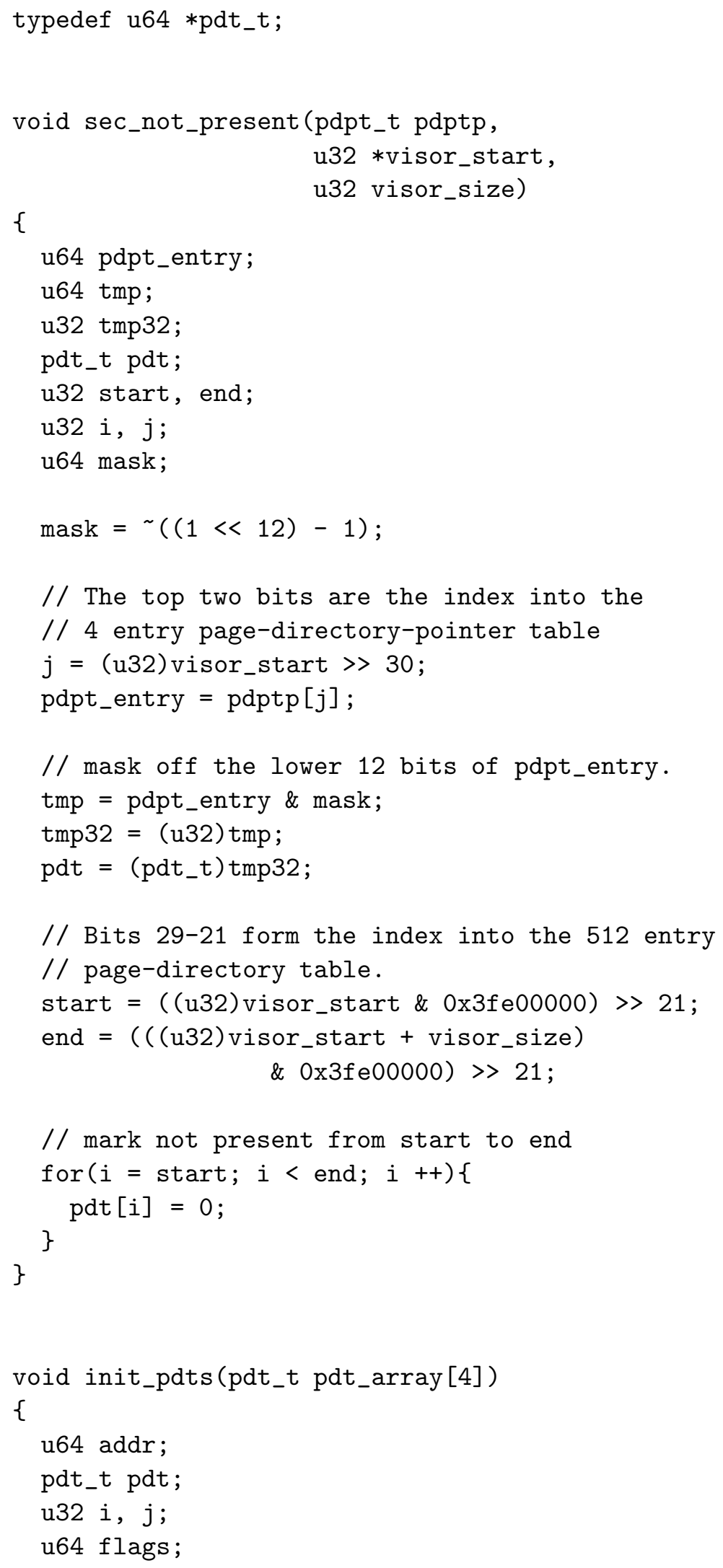




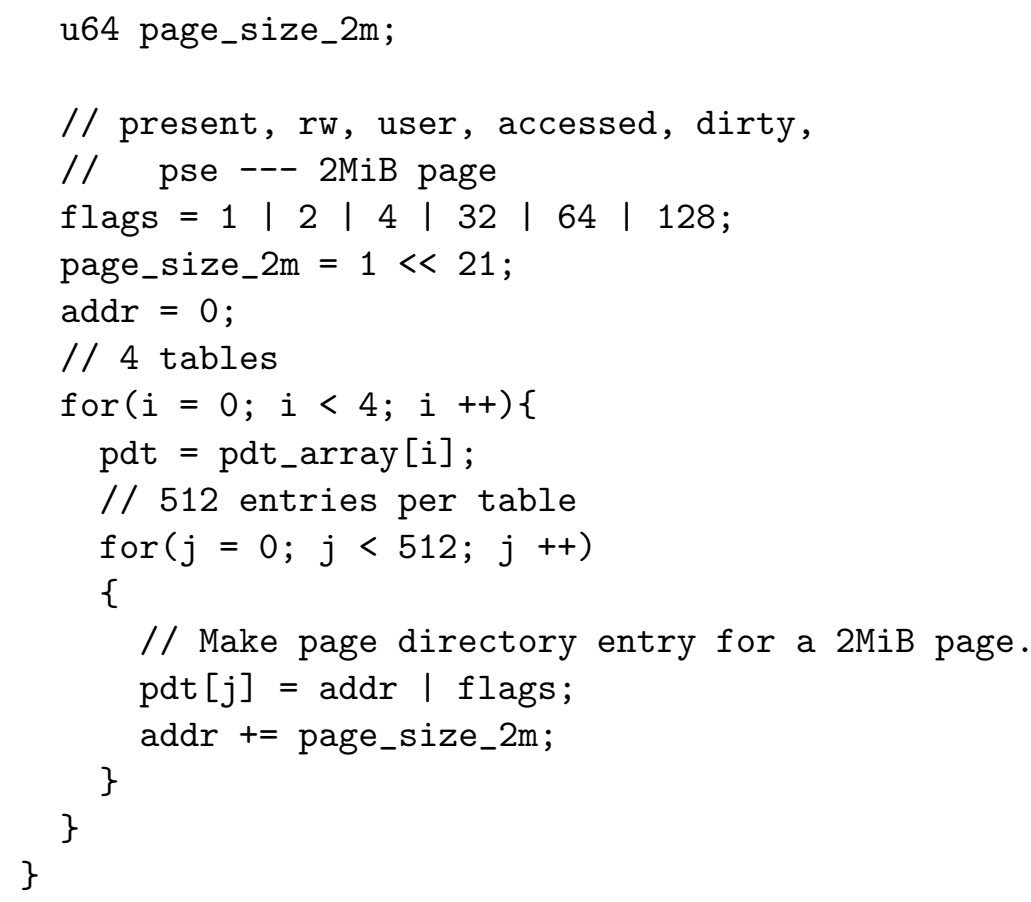




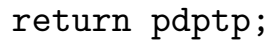

\section{B Y86++ Source Code for init_pdts}

We here give the Y86++ assembly corresponding to one of the above functions. init_pdts.

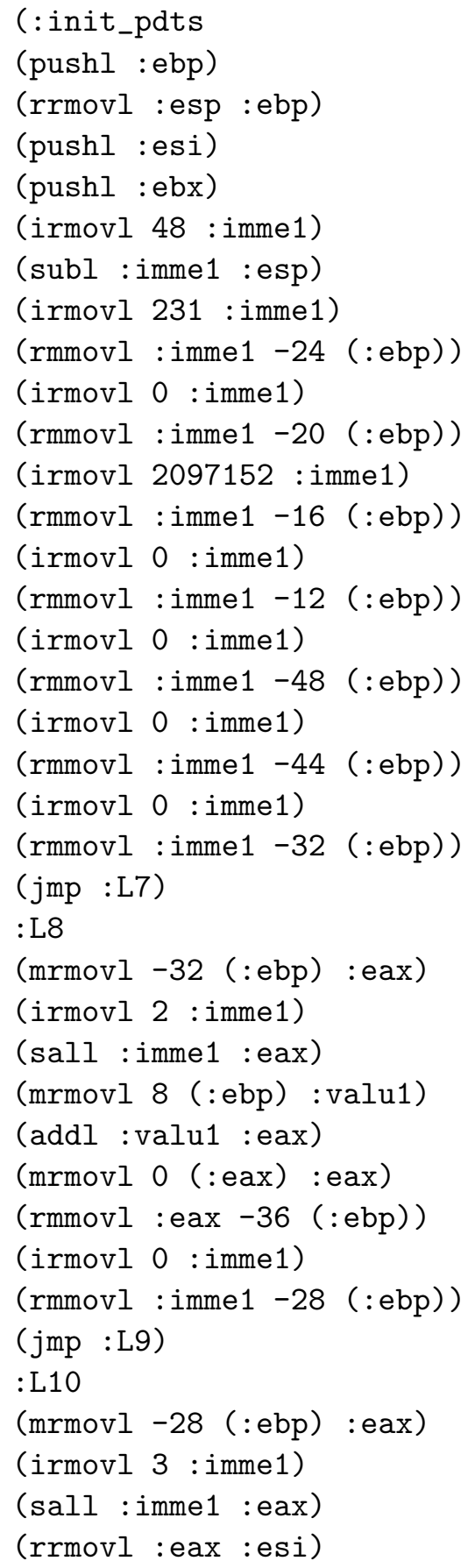




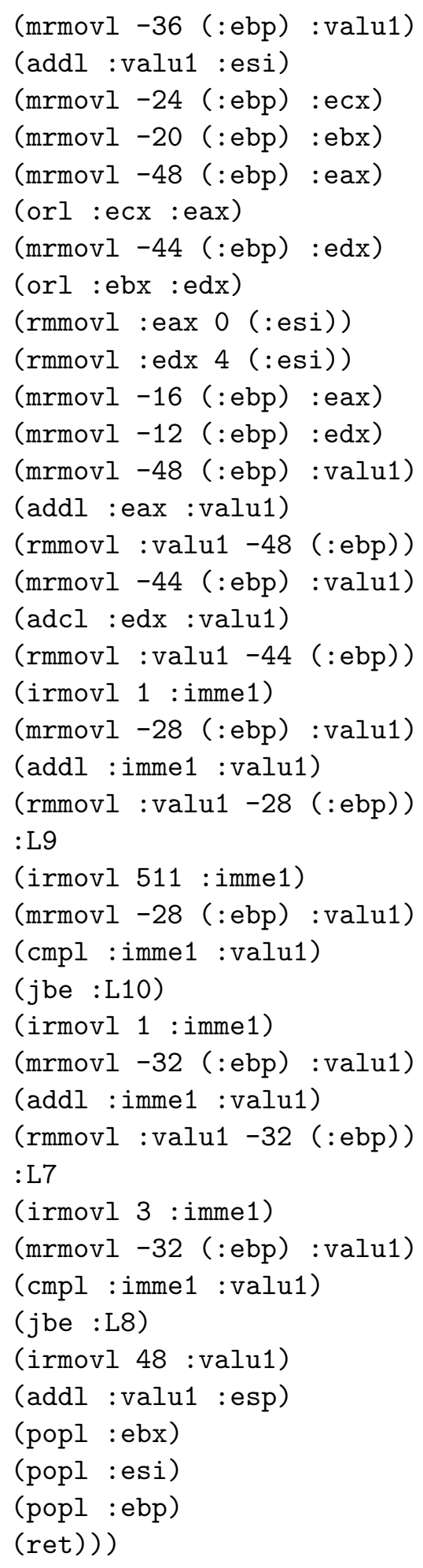




\section{References}

[1] Advanced Micro Devices (2005): AMD64 virtualization: Secure virtual machine architecture reference manual. AMD Publication no. 33047 rev. 3.01.

[2] E. Alkassar \& W. Paul (2008): On the verification of a "baby" hypervisor for a RISC machine; draft 0. available online.

[3] Paul Barham, Boris Dragovic, Keir Fraser, Steven Hand, Tim Harris, Alex Ho, Rolf Neugebauer, Ian Pratt $\&$ Andrew Warfield (2003): Xen and the art of virtualization. In: SOSP '03: Proceedings of the Nineteenth ACM Symposium on Operating Systems Principles, ACM, New York, NY, USA, pp. 164-177, doi:10.1145/ 945445.945462.

[4] William R. Bevier, Warren A. Hunt, Jr., J S. Moore \& William D. Young (1989): An Approach to System Verification. Journal of Automated Reasoning 5(4), pp. 411-428.

[5] Sven Beyer, Christian Jacobi, Daniel Kroning, Dirk Leinenbach \& Wolfgang J. Paul (2006): Putting it all together: Formal verification of the VAMP. International Journal on Software Tools Technology Transfer 8(4), pp. 411-430, doi:10.1007/s10009-006-0204-6.

[6] Randal E. Bryant \& David O'Hallaron (2003): Computer Systems: A Programmer's Perspective. PrenticeHall, Upper Saddle River, N.J.

[7] Ernie Cohen, Markus Dahlweid, Mark Hillebrand, Dirk Leinenbach, Michal Moskal, Thomas Santen, Wolfram Schulte \& Stephan Tobies (2009): VCC: A Practical System for Verifying Concurrent C. In: Proceedings of the 22nd International Conference on Theorem Proving in Higher Order Logics, TPHOLs '09, SpringerVerlag, Berlin, Heidelberg, pp. 23-42, doi:10.1007/978-3-642-03359-9_2.

[8] Intel Corporation (1999): Preboot Execution Environment, Verion 2.1. Technical Report, Intel Corporation.

[9] Jason Franklin, Arvind Seshadri, Ning Qu, Sagar Chaki \& Anupam Datta (2008): Attacking, Repairing, and Verifying SecVisor: A Retrospective on the Security of a Hypervisor. submitted to 8th USENIX Symposium on Operating Systems Design and Implementation (OSDI'08).

[10] David S. Hardin, Eric W. Smith \& William D. Young (2006): A Robust Machine Code Proof Framework for Highly Secure Applications. In: Proceeding of the 2006 International Workshop on ACL2, ACM.

[11] Kenneth Hess \& Amy Newman (2010): Practical Virtualization Solutions. Pearson Education, Boston.

[12] Sarah Hoffmann (2003): Formalising PC Hardware: A Model of the x86 Architecture. Master's thesis, Technische Universitat Dresden.

[13] Intel Corporation (2006): LaGrande technology preliminary architecture specification. Intel Publication no. D52212.

[14] (2006): JOS Operating System. http://pdos.csail.mit.edu/6.828/2006/overview.html.

[15] M. Kaufmann, P. Manolios \& J Moore (2000): Computer-Aided Reasoning: An Approach. Kluwer Academic Press, Boston, doi:10.1007/978-1-4615-4449-4.

[16] Gerwin Klein, Kevin Elphinstone, Gernot Heiser, June Andronick, David Cock, Philip Derrin, Dhammika Elkaduwe, Kai Engelhardt, Rafal Kolanski, Michael Norrish, Thomas Sewell, Harvey Tuch \& Simon Winwood (2009): seL4: formal verification of an OS kernel. In: Proceedings of the ACM SIGOPS 22nd symposium on Operating systems principles, SOSP '09, ACM, New York, pp. 207-220, doi:10.1145/1743546. 1743574 .

[17] KVM (2011): Kernel Based Virtual Machine. www.linux-kvm.org/page/Main_Page.

[18] Rebekah Leslie, Levent Erkök \& Flemming Andersen (2007): Formalizing Information Flow in a Haskell Hypervisor. In: Microkernels and Embedded Systems Workshop, MIKES'07.

[19] J. Matthews, J S. Moore, S. Ray \& D. Vroon (2006): Verification Condition Generation Via Theorem Proving. In M. Hermann \& A. Voronkov, editors: Proceedings of the 13th International Conference on Logic for Programming, Artificial Intelligence, and Reasoning (LPAR 2006), LNCS 4246, Springer, Phnom Penh, Cambodia, pp. 362-376, doi:10.1.1.101.503. 
[20] J. McDermott, J. Kirby, B. Montrose, T. Johnson \& M. Kang (2008): Re-engineering Xen internals for higher-assurance security. Information Security Technical Report 13(1), pp. 17-24, doi:10.1016/j.istr . 2008.01 .001$.

[21] John McDermott \& Leo Freitas (2008): A formal security policy for Xenon. In: FMSE '08: Proceedings of the 6th ACM workshop on Formal methods in security engineering, ACM, New York, NY, USA, pp. 43-52, doi:10.1145/1456396.1456401.

[22] J S. Moore (2003): Inductive Assertions and Operational Semantics. In: CHARME 2003, Volume 2860 of LNCS, Springer-Verlag, pp. 289-303, doi:10.1007/s10009-005-0180-2.

[23] Sandip Ray (2005): Using Theorem Proving and Algorithmic Design Procedures for Large-Scale System Verification. Ph.D. thesis, University of Texas at Austin.

[24] A. Roscoe, J. Woodcock \& L. Wulf (1997): Non-interference through nondeterminism. In: Proceedings ESORICS, pp. 33-52.

[25] Arvind Seshadri, Mark Luk, Ning Qu \& Adrian Perrig (2007): SecVisor: A Tiny Hypervisor to Provide Lifetime Kernel Code Integrity for Commodity OSes. In: SOSP '07: Proceedings of Twenty-first ACM SIGOPS Symposium on Operating Systems Principles, ACM, pp. 335-350, doi:10.1145/1323293.1294294.

[26] James E. Smith \& Ravi Nair (2005): Virtual Machines: Versatile Platforms for Systems and Processes. Morgan Kaufmann, Boston.

[27] Hendrik Tews, Bart Jacobs, Erik Poll, Marko van Eekelen \& Peter van Rossum (2005): Specification and Verification of the Nova microhypervisor. Project Robin deliverable D.6, Radboud University Nijmegen.

[28] Verisoft (2008): Verisoft Repository. www.verisoft.de/VerisoftRepository.html. 